\title{
Increases in urinary albumin and beta2-microglobulin are independently associated with blood pressure in the Japanese general population: the Takahata Study
}

\author{
Yusuke Mashima $^{1}$, Tsuneo Konta ${ }^{1}, K$ Kosuke Kudo ${ }^{1}$, Satoshi Takasaki ${ }^{1}, K$ Kazunobu Ichikawa ${ }^{1}, K$ Kazuko Suzuki ${ }^{1}$, \\ Yoko Shibata ${ }^{1}$, Tetsu Watanabe ${ }^{1}$, Takeo Kato ${ }^{2}$, Sumio Kawata ${ }^{3}$ and Isao Kubota ${ }^{1}$
}

Essential hypertension is a multifactorial disorder and a risk factor for renal failure and cardiovascular disease. Recently it was hypothesized that subtle acquired renal injury such as renal microvascular and tubulointerstitial damage induces salt-sensitive hypertension. The objective of this study was to examine the relationship between blood pressure and renal abnormalities in the Japanese general population. The participants in this community-based, cross-sectional study were 1,965 subjects over 40 years old, without renal insufficiency and antihypertensive medication. Urine albumin-creatinine ratio (UACR) and beta2microglobulin-creatinine ratio (UBCR) were measured in single spot urine samples, as markers of renal microvascular and tubulointerstitial damage, respectively. Multiple linear regression analysis showed a significant positive correlation of blood pressure with UACR and UBCR, but not with estimated glomerular filtration rate. In multiple logistic regression analysis, the increases in UACR and UBCR were independently associated with hypertension, after adjustment for possible confounders. Higher levels of UACR $\left(\geqslant 5.9 \mathrm{mg} \mathrm{g}^{-1}\right)$ and UBCR $\left(\geqslant 145 \mu \mathrm{g} \mathrm{g}^{-1}\right)$ were associated with a significantly higher risk of hypertension, compared with UACR $\leqslant 5.8 \mathrm{mg} \mathrm{g}^{-1}$ and UBCR $\leqslant 84.5 \mu \mathrm{g} \mathrm{g}^{-1}$, respectively. Furthermore, there was a positive relationship between urinary sodium excretion and blood pressure in subjects with high UBCR tertile. This study showed that the increases in urinary albumin and beta2-microglobulin were independently associated with blood pressure in a general population. These renal abnormalities may be differentially related to the development of hypertension.

Hypertension Research (2011) 34, 831-835; doi:10.1038/hr.2011.42; published online 28 April 2011

Keywords: albuminuria; beta2-microglobulin; salt intake

\section{INTRODUCTION}

Hypertension is one of the most common chronic diseases in industrialized countries, and is a risk factor for end-stage renal disease and cardiovascular disease. A large number of studies indicate that hypertension is a complex disorder, and in the majority of subjects it is categorized as essential hypertension without an underlying cause. Several mechanisms have been proposed to explain the development of essential hypertension, including genetic background, ${ }^{1}$ congenital paucity of nephrons, ${ }^{2}$ with the involvement of the renin-angiotensin system, ${ }^{3}$ sympathetic nerve activity ${ }^{4}$ and salt intake. ${ }^{5}$ Recently Johnson et al. ${ }^{6}$ propounded a new hypothesis that subtle acquired renal injury, such as renal microvascular and tubulointerstitial damage, in the absence of clinical renal disease, may induce saltsensitive hypertension. According to this hypothesis, low-grade renal microvascular injury reduces renal blood flow and induces renal ischemia, resulting in increased tubular sodium reabsorption, tubu- lointerstitial injury and salt-sensitive hypertension. This hypothesis is very attractive and reasonable, and is based on clinical and experimental observations. ${ }^{6}$ However, it is not known whether renal microvascular and tubulointerstitial damage influences blood pressure in the general population.

It is difficult to directly evaluate renal microvascular and tubulointerstitial damage in an epidemiological study. However, urinary parameters such as albumin and beta2-microglobulin may be used as markers of renal microvascular and tubulointerstitial injury, as they reflect dysfunction of the glomerulus, a part of renal arterioles, and tubules, respectively. Previous studies have indicated that low-grade albuminuria is a risk factor for incident hypertension and increasing blood pressure. ${ }^{7,8}$ However, these studies did not investigate the involvement of tubulointerstitial damage. Therefore, a cross-sectional study was performed to clarify the relationship between blood pressure and renal microvascular and tubulointerstitial damage

\footnotetext{
${ }^{1}$ Department of Cardiology, Pulmonology and Nephrology, Yamagata University School of Medicine, Yamagata, Japan; ${ }^{2}$ Department of Neurology, Hematology, Metabolism, Endocrinology and Diabetes, Yamagata University School of Medicine, Yamagata, Japan and ${ }^{3}$ Department of Gastroenterology, Yamagata University School of Medicine, Yamagata, Japan

Correspondence: Dr T Konta, Department of Cardiology, Pulmonology and Nephrology, Yamagata University School of Medicine, Yamagata, 2-2-2, lida-Nishi, Yamagata 990-9585, Japan. 
in the general population, using urinary albumin and beta2-microglobulin as markers of these renal abnormalities.

\section{METHODS}

\section{Study population}

This study was part of the ongoing Molecular Epidemiological Study in the Regional Characteristics of 21st Century Centers of Excellence Program and the Global Centers of Excellence Program in Japan. Details of the study methodology have been described elsewhere. ${ }^{9}$

This study used a community-based annual health checkup that invited all inhabitants over the age of 40 years in Takahata town (total population 26 026), located in the northern part of Japan. This region has a resident population of 15222 adults over the age of 40 years ( 7109 males and 8113 females). From June 2004 to November 2005, 1394 men and 1771 women (total, 3165, aged from 40 to 87 years) took part in the program and agreed to join the study. The participation rate was $20.8 \%$. This study was approved by the Institutional Ethics Committee. All participants gave written informed consent.

Among the 3,165 participants, 57 subjects were excluded from the present analysis because their data were incomplete. In addition, 1,056 subjects using antihypertensive medication and 87 subjects with renal insufficiency (estimated glomerular filtration rate (eGFR) $<60 \mathrm{ml} \mathrm{min}^{-1}$ per $1.73 \mathrm{~m}^{2}$ ) were excluded to avoid confounding influence by antihypertensive drugs and advanced renal damage on the relationship between blood pressure and urinary excretion of albumin and beta2-microglobulin. Therefore, data for 896 males and 1069 females (a total of 1965 subjects) were used in the final statistical analysis.

\section{Measurements}

Subjects used a self-report questionnaire to document their medical history, current medications, family history, clinical symptoms, smoking habit (current smoker or non-smoker) and alcohol intake (current drinker or non-drinker). Systolic and diastolic blood pressures were determined using a mercury manometer, with subjects in the sitting position and having rested for at least $5 \mathrm{~min}$. Blood pressures were measured twice, and the mean value was used in statistical analyses. Hypertension was defined as a systolic blood pressure $\geqslant 140 \mathrm{~mm} \mathrm{Hg}$ and/or diastolic blood pressure $\geqslant 90 \mathrm{~mm} \mathrm{Hg}$. Body mass index (BMI) was calculated as weight $(\mathrm{kg})$ divided by height squared $\left(\mathrm{m}^{2}\right)$. For both men and women, obesity was defined as BMI $\geqslant 25.0 \mathrm{~kg} \mathrm{~m}^{-2}$. Subjects with diabetes were identified either by self-reported physical diagnosis or by a fasting plasma glucose concentration $\geqslant 126 \mathrm{mg} \mathrm{dl}^{-1}$ or hemoglobin Alc value $\geqslant 6.5 \%$.

Serum uric acid, triglyceride, total cholesterol and low-density lipoprotein cholesterol concentrations were measured by enzymatic methods. High-density lipoprotein cholesterol concentration was measured directly. Serum total protein was measured by the colorimetric Biuret method. Plasma glucose levels were measured by the hexokinase enzymatic reference method. Urinary albumin concentration was determined by immunoturbidimetry (BML, Tokyo, Japan). Urinary beta2-microglobulin concentration was determined by latex agglutination method (BML). The urine albumin-creatinine ratio (UACR) and urine beta2-microglobulin-creatinine ratio (UBCR) were measured in single spot urine specimens collected in the morning between 0730 and $0930 \mathrm{~h}$ before breakfast. Albuminuria was defined as UACR $\geqslant 30 \mathrm{mgg}^{-1} \cdot{ }^{10}$ Serum creatinine level was measured by an enzymatic method and GFR was estimated using the Japanese version of the modified MDRD equation. ${ }^{11}$ Twenty-four-hour urinary sodium and potassium excretion were estimated in a spot urine specimen, using Kawasaki's equation. ${ }^{12}$ We used the tertiles of UACR and UBCR to examine the relationship between salt intake and blood pressure.

\section{Statistical analyses}

The Student's $t$-test was used to compare the mean values. To examine the correlation between blood pressure and various parameters, including eGFR, UACR and UBCR, simple and multiple linear regression analyses were performed. The parameters included in these analyses were gender, age, BMI, uric acid, hemoglobin and total protein concentrations, hemoglobin Alc, total cholesterol, triglyceride and high-density lipoprotein cholesterol concentrations, smoking, alcohol consumption, and estimated 24-h urinary sodium and potassium excretion. As UACR and UBCR were positively skewed, the values were logarithmically transformed ( $\log$ UACR and $\log$ UBCR) before the correlation analyses were performed. To test whether UACR and UBCR were independently associated with hypertension, multivariate logistic regression analysis was performed, with adjustment of the model for possible confounders, including gender, age, BMI, uric acid, hemoglobin and total protein concentrations, hemoglobin Alc, total cholesterol, triglyceride and high-density lipoprotein cholesterol concentrations, smoking, alcohol consumption, eGFR, and estimated 24-h urinary sodium and potassium excretion. Continuous data are expressed as mean \pm s.d. and data with a skewed distribution are presented as medians with interquartile range. All statistical analyses were performed using StatView version 5 software (SAS Institute, Cary, NC, USA). A significant difference was defined as $P<0.05$.

\section{RESULTS}

\section{Characteristics of the subjects}

The baseline characteristics of the 1965 subjects for whom data were entered into the final analysis are shown in Table 1 . The overall mean age was 60 years. There were 896 men (45.6\%), 619 subjects (31.5\%) with hypertension, 500 subjects $(25.4 \%)$ were obese, 117 subjects (6.0\%) had diabetes and 291 (14.8\%) subjects had albuminuria. Average 24-h urinary sodium and potassium excretion, as estimated by Kawasaki's formula, was $216.9 \mathrm{mEq}$ per day and $57.2 \mathrm{mEq}$ per day, respectively. Compared with the normotensive subjects, the hypertensive subjects were older, more likely to be males and showed significantly higher values in clinical parameters including BMI, uric acid, hemoglobin, hemoglobin A1c, triglyceride, urine sodium excretion, UACR and UBCR but not eGFR.

\section{Correlation between blood pressure and renal parameters}

The correlation of blood pressure with UACR and UBCR was first examined by simple linear regression analysis. The UACR and UBCR

Table 1 Baseline characteristics of the subjects who were included in the study

\begin{tabular}{|c|c|c|c|}
\hline & Total subjects & Normotensive & Hypertensive \\
\hline Number & 1965 & 1346 & 619 \\
\hline Male gender (\%) & 45.6 & 40.9 & $55.7^{*}$ \\
\hline Age (years) & $60.1 \pm 10.2$ & $59.1 \pm 10.3$ & $62.4 \pm 9.6^{*}$ \\
\hline Systolic BP (mm Hg) & $129.8 \pm 15.5$ & $122.2 \pm 11.3$ & $146.4 \pm 9.2^{*}$ \\
\hline Diastolic BP (mm Hg) & $77.9 \pm 10.2$ & $73.8 \pm 8.2$ & $86.9 \pm 8.2^{*}$ \\
\hline Body mass index $\left(\mathrm{kg} \mathrm{m}^{-2}\right)$ & $23.1 \pm 3.1$ & $22.6 \pm 3.0$ & $24.1 \pm 3.0^{*}$ \\
\hline Serum creatinine $\left(\mathrm{mg} \mathrm{dl}^{-1}\right)$ & $0.65 \pm 0.13$ & $0.65 \pm 0.13$ & $0.66 \pm 0.13^{*}$ \\
\hline eGFR $\left(\mathrm{ml} \mathrm{min}-1\right.$ per $\left.1.73 \mathrm{~m}^{2}\right)$ & $84.7 \pm 14.6$ & $84.7 \pm 14.8$ & $84.8 \pm 14.4$ \\
\hline Uric acid (mg dl $\left.{ }^{-1}\right)$ & $4.92 \pm 1.31$ & $4.80 \pm 1.27$ & $5.18 \pm 1.34^{*}$ \\
\hline Hemoglobin $\left(\mathrm{g} \mathrm{dl}^{-1}\right)$ & $13.8 \pm 1.5$ & $13.6 \pm 1.5$ & $14.1 \pm 1.5^{*}$ \\
\hline Hemoglobin Alc (\%) & $5.20 \pm 0.69$ & $5.18 \pm 0.68$ & $5.25 \pm 0.70^{*}$ \\
\hline Triglyceride $\left(\mathrm{mg} \mathrm{dl}^{-1}\right)$ & $105.4 \pm 67.3$ & $99.3 \pm 59.1$ & $118.6 \pm 80.9^{*}$ \\
\hline $\mathrm{HDL}-\mathrm{C}\left(\mathrm{mg} \mathrm{dl}^{-1}\right)$ & $59.4 \pm 14.6$ & $60.1 \pm 14.7$ & $58.0 \pm 14.3^{*}$ \\
\hline e24hUNa (mEq per day) & $216.9 \pm 57.4$ & $212.0 \pm 56.1$ & $227.0 \pm 58.7^{*}$ \\
\hline e24hUK (mEq per day) & $57.2 \pm 11.8$ & $57.1 \pm 11.9$ & $57.5 \pm 11.4$ \\
\hline Obese (\%) & 25.4 & 21.1 & $34.9^{*}$ \\
\hline Smoker (\%) & 35.6 & 35.2 & 36.3 \\
\hline Drinker (\%) & 42.4 & 40.0 & $47.7^{*}$ \\
\hline Diabetes (\%) & 6.0 & 5.4 & 7.1 \\
\hline Albuminuria (\%) & 14.8 & 11.7 & $21.5^{*}$ \\
\hline UACR ( $\left.\mathrm{mgg}^{-1}\right)$ & $8.8(4.7-17.9)$ & $7.7(4.3-15.1)$ & $11.6(5.9-26.7)^{*}$ \\
\hline UBCR $\left(\mu g^{-1}\right)$ & $108(72.0-175)$ & $102(68.4-159)$ & $128(79.6-212)^{*}$ \\
\hline
\end{tabular}

Abbreviations: BP, blood pressure; eGFR, estimated glomerular filtration rate; e24hUNa, estimated 24-h urinary sodium excretion; e24hUK, estimated 24-h urinary potassium excretion; HDL-C, high-density lipoprotein cholesterol; UACR, urine albumin-creatinine ratio; UBCR, urine beta2-microglobulin-creatinine ratio.

UACR and UBCR are expressed as medians with (25th-75th quartiles). ${ }^{*} P<0.05$ normotensive vs. hypertensive. 

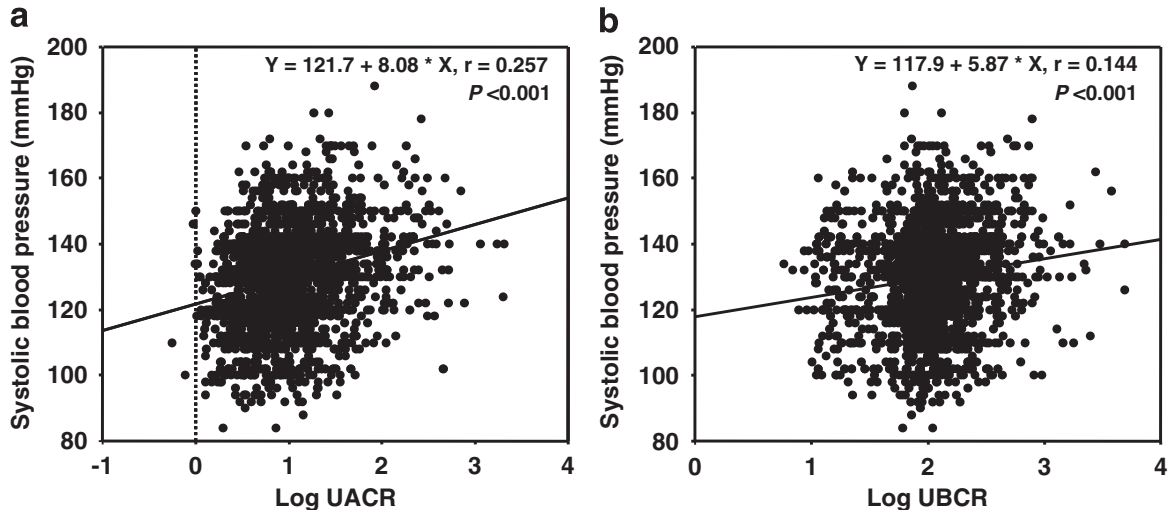

Figure 1 The correlation between urine albumin-creatinine ratio (UACR) (a), urine beta2-microglobulin-creatinine ratio (UBCR) (b) and systolic blood pressure. log UACR, logarithmically transformed UACR; log UBCR, logarithmically transformed UBCR.

distributions were highly skewed toward larger values; therefore logarithmically transformed UACR and UBCR values were used in these analyses. There was a significant positive correlation of systolic blood pressure with log UACR (Figure 1a; $y=121.7+8.08 \mathrm{x}, r=0.257$, $P<0.001$ ), and $\log$ UBCR (Figure $1 b, y=117.8+5.87 \mathrm{x}, r=0.144$, $P<0.001)$. Similar positive correlations were observed for diastolic blood pressure with $\log$ UACR $(y=75.1+2.82 \mathrm{x}, r=0.137, P<0.001)$, and $\log$ UBCR $(y=72.1+2.86 \mathrm{x}, r=0.107, P<0.001)$.

Relationship of blood pressure with UACR and UBCR was then evaluated by simple and multiple linear regression analyses. As shown in Table 2, there were significant positive correlations of systolic blood pressure with renal parameters such as log UACR (coefficient 5.78, $P<0.001$ ) and $\log$ UBCR (coefficient 2.30, $P=0.013$ ) but not with eGFR (coefficient 0.02, $P=0.517$ ). Beside UACR and UBCR, systolic blood pressure was correlated positively with age, male gender, BMI, uric acid, triglyceride, high-density lipoprotein cholesterol and hemoglobin, and negatively correlated with smoking and urine potassium excretion. Again, similar positive correlations were observed for diastolic blood pressure with $\log$ UACR (coefficient 2.77, $P<0.001$ ) and $\log$ UBCR (coefficient 2.17, $P<0.001$ ). These results suggest that UACR and UBCR are independently associated with blood pressure.

\section{Renal factors associated with hypertension}

Multiple logistic regression analysis was performed to examine the quantitative relationship of UACR and UBCR with hypertension (Table 3). UACR was independently associated with hypertension after adjustment for possible confounders, and higher UACR levels (5.9-13.6 $\left.\mathrm{mg} \mathrm{g}^{-1}\right)$ were associated with an increased risk of hypertension compared with a UACR $\leqslant 5.8 \mathrm{mgg}^{-1}$ (odds ratio $1.39,95 \%$ confidence interval $1.04-1.86, P=0.027$ ). The odds ratio was further increased for higher values of UACR. Similarly, UBCR was independently associated with hypertension, and higher UBCR levels $\left(\geqslant 145 \mu \mathrm{gg}^{-1}\right)$ was associated with an increased risk of hypertension compared with a UBCR $\leqslant 84.5 \mu \mathrm{gg}^{-1}$ (odds ratio $1.67,95 \%$ confidence interval 1.26-2.20, $P<0.001$ ). These results indicate that subtle increases in UACR and UBCR are independent risk factors for hypertension.

\section{Influence of UACR and UBCR on the relationship between salt} intake and blood pressure

In all subjects, multiple linear regression analysis showed that the correlation between systolic blood pressure and urine sodium excretion was nonsignificant; then we investigated whether UACR and UBCR influenced the relationship between salt intake and blood
Table 2 Linear regression coefficients for the association of systolic blood pressure with renal parameters ${ }^{a}$

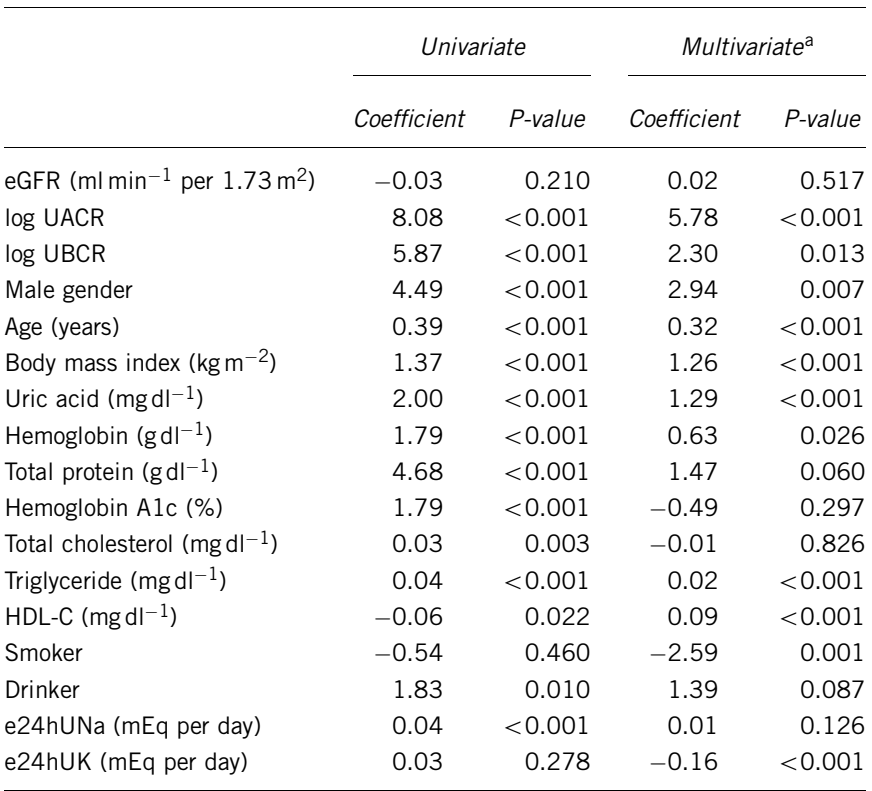

Abbreviations: HDL-C, high-density lipoprotein cholesterol; eGFR, estimated glomerular filtration rate; e24hUK, estimated 24-h urinary potassium excretion; e24hUNa, estimated 24-h urinary sodium excretion; log UACR, logarithmically transformed urine albumin-creatinine ratio; log UBCR, logarithmically transformed urine beta2-microglobulin-creatinine ratio.

${ }^{a}$ Adjusted for gender, age, body mass index, uric acid, hemoglobin and total protein concentrations, $\mathrm{HbAlc}$, total cholesterol, triglyceride and $\mathrm{HDL}$-cholesterol concentrations, smoking, alcohol consumption, and estimated 24 -h urinary sodium and potassium excretion.

pressure. Subjects were divided according to the tertiles of UACR $\left(\leqslant 5.8,5.9-13.6, \geqslant 13.7 \mathrm{mgg}^{-1}\right)$ and UBCR ( $\leqslant 84.5,84.6-144$, $\left.\geqslant 145 \mu \mathrm{gg}^{-1}\right)$, and linear regression analyses were performed in each UACR and UBCR tertile. Systolic blood pressure was significantly increased with increasing tertiles of UACR $(P<0.001)$ and UBCR $(P<0.001)$. The subgroup analyses using multivariate model disclosed that the relation was nonsignificant in hypertensive $(N=619)$, normotensive subjects $(N=1346)$ and subjects with all tertiles of UACR, that is, low and middle tertiles of UBCR. Only the subjects with high UBCR showed a significant positive relation between systolic blood pressure and urine sodium excretion $(P=0.012)$ (Table 4$)$.

These results indicate that the effects of UACR and UBCR on the relationship between salt intake and blood pressure seems to be different, and high UBCR might be related to the salt-dependent blood pressure increase. 
Table 3 Multiple logistic regression model for factors predicting hypertension $^{\mathrm{a}}$

\begin{tabular}{lccr}
\hline & Odds ratio & $(95 \% \mathrm{Cl})$ & P-value \\
\hline UACR & & & \\
$\leqslant 5.8 \mathrm{mgg}^{-1}$ & 1.00 & Reference & \\
$5.9-13.6 \mathrm{mgg}^{-1}$ & 1.39 & $(1.04,1.86)$ & 0.027 \\
$\geqslant 13.7 \mathrm{mgg}^{-1}$ & 2.06 & $(1.50,2.82)$ & $<0.00$
\end{tabular}

$U B C R$

$\begin{array}{llcr}\leqslant 84.5 \mu \mathrm{gg}^{-1} & 1.00 & \text { Reference } & \\ 84.6-144 \mu \mathrm{gg}^{-1} & 1.27 & (0.97,1.67) & 0.083 \\ \geqslant 145 \mu \mathrm{gg}^{-1} & 1.67 & (1.26,2.20) & <0.001\end{array}$

Abbreviations: $\mathrm{BP}$, blood pressure; $\mathrm{Cl}$, confidence interval; UACR, urine albumin-creatinine ratio; UBCR, urine beta2-microglobulin-creatinine ratio.

aAdjusted for gender, age, body mass index, uric acid,

concentrations, $\mathrm{HbAlc}$, total cholesterol, triglyceride and $\mathrm{HDL}$-cholesterol concentrations,

smoking, alcohol consumption, estimated 24-h urinary sodium and potassium excretion and estimated glomerular filtration rate.

Table 4 Linear regression coefficients for the association of systolic blood pressure with urine sodium excretion in subgroups

\begin{tabular}{|c|c|c|c|c|}
\hline & \multicolumn{2}{|c|}{ Univariate } & \multicolumn{2}{|c|}{ Multivariate $^{a}$} \\
\hline & Coefficient & $\mathrm{P}$-value & Coefficient & P-value \\
\hline Hypertensive ( $N=619)$ & 0.012 & 0.052 & -0.002 & 0.780 \\
\hline Normotensive $(N=1346)$ & 0.017 & 0.003 & 0.012 & 0.084 \\
\hline \multicolumn{5}{|l|}{ UACR } \\
\hline$\leqslant 5.8 \mathrm{mgg}^{-1}(N=655)$ & 0.028 & 0.013 & 0.007 & 0.628 \\
\hline $5.9-13.6 \mathrm{mgg}^{-1}(N=650)$ & 0.015 & 0.176 & 0.008 & 0.531 \\
\hline$\geqslant 13.7 \mathrm{mgg}^{-1}(N=660)$ & 0.014 & 0.213 & 0.017 & 0.174 \\
\hline \multicolumn{5}{|l|}{$U B C R$} \\
\hline$\leqslant 84.5 \mu \mathrm{gg}^{-1}(N=654)$ & 0.031 & 0.006 & 0.007 & 0.616 \\
\hline $84.6-144 \mu g^{-1}(N=655)$ & 0.025 & 0.022 & -0.002 & 0.886 \\
\hline$\geqslant 145 \mu \mathrm{gg}^{-1}(N=656)$ & 0.034 & 0.001 & 0.030 & 0.012 \\
\hline
\end{tabular}

Abbreviations: UACR, urine albumin-creatinine ratio; UBCR, urine beta2-microglobulincreatinine ratio.

${ }^{a}$ Adjusted for gender, age, body mass index, uric acid, hemoglobin and total protein concentrations, $\mathrm{HbAlc}$, total cholesterol, triglyceride and $\mathrm{HDL}$-cholesterol concentrations, smoking, alcohol consumption, and estimated 24-h urinary potassium excretion.

\section{DISCUSSION}

This study showed that UACR and UBCR were independently associated with blood pressure, and small increases in UACR and UBCR were risk factors for hypertension in the general Japanese population. Furthermore, the relationship between salt intake and blood pressure might be modified by UBCR levels.

A previous study by Wang et al. ${ }^{7}$ demonstrated that low-grade albuminuria ( $>6.66 \mathrm{mgg}^{-1}$ in men, $>15.24 \mathrm{mgg}^{-1}$ in women) is a risk factor for hypertension and blood pressure progression. Forman et al. ${ }^{8}$ reported that a small increase in UACR within the normal range (3.68-23.84 $\mathrm{mgg}^{-1}$ in young women, $4.34-24.17 \mathrm{mgg}^{-1}$ in older women) predicted incident hypertension in non-diabetic females. The present findings seem to be in agreement with these previous reports, and suggest that very low-grade albuminuria $\left(\geqslant 5.9 \mathrm{mg} \mathrm{g}^{-1}\right)$ may affect blood pressure, independent of salt intake and tubulointerstitial damage.

Although it has been reported that a high UBCR $\left(\geqslant 1000 \mu \mathrm{gg}^{-1}\right)$ was associated with high all-cause mortality in a cadmium-polluted area, ${ }^{13}$ the relationship between tubulointerstitial damage and blood pressure has not been examined in a cohort study. To our knowledge, this is the first report that occult tubulointerstitial damage (UBCR $\geqslant 145 \mu \mathrm{gg}^{-1}$ ) is independently associated with hypertension in the general population. Generally, urinary beta2-microglobulin is not measured during community-based health checks. However, on the basis of present findings, the measurement of urinary beta2-microglobulin may help in screening for subjects with a high risk of cardiovascular disease.

Interestingly, in this study, the effects of UACR and UBCR on the development of hypertension were independent, and there was a positive relationship between urinary sodium excretion and blood pressure in subjects with high UBCRs. This suggests that glomerular and tubulointerstitial damages have differential effects on blood pressure. Johnson et al. ${ }^{6}$ hypothesized that renal microvascular and tubulointerstitial damage may induce salt-sensitive hypertension. This study showed that the effect of salt on blood pressure is related with UBCR levels but not with UACR or blood pressure levels. This finding suggests that a subtle tubular injury might cause the salt-sensitive hypertension, in agreement with a hypothesis proposed by Johnson et al. ${ }^{6}$

In contrast, the present finding that high urinary sodium excretion did not significantly increase blood pressure in subjects with elevated UACRs seems to be inconsistent with the hypothesis. It has been reported that the prevalence of hypertension was higher in subjects with glomerular disease than in those with tubulointerstitial disease. ${ }^{14}$ These findings suggest that albuminuria itself may elevate blood pressure, irrespective of sodium intake or tubulointerstitial damage. It has been documented that glomerular hypertension induces sodium sensitivity and microalbuminuria. This raises a possibility that glomerular hypertension might be an underlying mechanism in the development of albuminuria and salt-sensitive hypertension, and the albuminuria-related blood pressure increase might precede saltsensitive hypertension.

In patients with renal disease, a decline in GFR is one of the most important factors contributing to the development of hypertension. ${ }^{14}$ However, in this study, there was no correlation between blood pressure and eGFR. This suggests that GFR may not have an important role in the regulation of blood pressure in subjects without renal insufficiency.

The clinical implications of the present findings are that higher levels of urinary albumin (UACR $\geqslant 5.9 \mathrm{mg} \mathrm{g}^{-1}$ ) and beta2-microglobulin (UBCR $\geqslant 145 \mu \mathrm{gg}^{-1}$ ) are prevalent in the general population, and should be regarded as risk factors for the future development of hypertension and hypertension-related complications, including chronic kidney disease and cardiovascular disease. It is expected that early detection and treatment of hypertension can prevent the progression of renal damages and salt-sensitive hypertension. Although it is not known how to reverse tubulointerstitial damage, previous studies advocated several measures to attenuate albuminuria, including salt-intake restriction, weight loss, normalizing plasma glucose levels, usage of renin-angiotensin system inhibitor and lowering blood pressure. ${ }^{15}$ These interventions might be recommended in subjects with albuminuria. Therefore, it is worthwhile identifying these abnormalities at their earliest stages in clinical practice.

This study has several limitations that should be noted. First, because this was a cross-sectional study, it is difficult to infer causality between renal damage and blood pressure. To clarify the causal relationship between albuminuria, tubulointerstitial damages and hypertension, a longitudinal cohort study that examines the incident hypertension in normotensive population would be required. Some reports indicated that hypertension was a risk factor for albuminuria, ${ }^{16}$ whereas others showed that albuminuria is a risk factor for hypertension. ${ }^{7,8}$ On the basis of these observations, it might be 
speculated that the relationship between blood pressure and renal damage is bidirectional, and forms a vicious circle in the progression of chronic kidney disease and hypertension. Second, blood pressure, UACR and UBCR were measured on one occasion. It is well known that these parameters show high intra-individual variation. ${ }^{17}$ The one measurement of urine sample might have led to some misclassification of the category of UACR and UBCR. Such misclassification would probably have been nondifferential and would have biased the relationship toward the null. Therefore, there is a possibility that the observed relationship between blood pressure and renal parameters may have been underestimated. In addition, urine albumin excretion is affected by various factors. There is a possibility that the increase in UACR is induced by not glomerular damage but by other factors such as glomerular hypertension and insulin resistance in some subjects. Third, the subjects were volunteers, not randomly assigned people and we excluded the subjects receiving antihypertensive treatment and those with renal insufficiency, to avoid the confounding effects of antihypertensive drugs and renal function on the relationships between blood pressure, UACR, UBCR and urinary sodium excretion. Therefore, selection of the study population was biased, and caution is required in generalizing the findings to the whole population. Fourth, the ideal evaluation of salt sensitivity is by giving varying salt loads to the participants and assessing their blood pressure. However this method is not feasible in the epidemiological setting. In this study we compared the blood pressures between different urine sodium excretion subgroups. There might be a discrepancy between urine excretion of sodium and the amount of salt intake. To estimate the 24$\mathrm{h}$ urine sodium excretion, the original study by Kawasaki ${ }^{12}$ used the urine collected at approximately $0800 \mathrm{~h}$ before breakfast as a second morning voiding urine. In the current study urine samples were collected between 0730 to $0930 \mathrm{~h}$ before breakfast. Although the timing of urine sampling was not exactly the same as the original method, it seems to be acceptable to use Kawasaki's equation for the urine sodium excretion in this study. Considering these limitations, the result should be interpreted with a caution.

In conclusion, this study showed that low-grade glomerular and tubulointerstitial damage might be independently associated with the increase in blood pressure in the general Japanese population. The precise mechanism by which these abnormalities influence blood pressure requires clarification, in order to prevent further increases in the prevalence of hypertension and chronic kidney disease.

\section{CONFLICT OF INTEREST}

The authors declare no conflict of interest.

\section{ACKNOWLEDGEMENTS}

This study was supported by a grant-in-aid from the 21st Century Center of Excellence (COE) and the Global COE Program of the Japan Society for the Promotion of Science.

1 Lifton RP. Molecular genetics of human blood pressure variation. Science 1996; 272 : 676-680.

2 Brenner BM, Garcia DL, Anderson S. Glomeruli and blood pressure. Less of one, more the other? Am J Hypertens 1988; 1: 335-347.

3 Laragh $\mathrm{JH}$. Renin-angiotensin-aldosterone system for blood pressure and electrolyte homeostasis and its involvement in hypertension, in congestive heart failure and in associated cardiovascular damage (myocardial infarction and stroke). J Hum Hypertens 1995; 9: 385-390.

4 Tuck ML. The sympathetic nervous system in essential hypertension. Am Heart J 1986; 112: 877-886.

5 Stamler J, Rose G, Elliott P, Dyer A, Marmot M, Kesteloot H, Stamler R. Findings of the International Cooperative INTERSALT Study. Hypertension 1991; 17: I9-I15.

6 Johnson RJ, Rodriguez-Iturbe B, Nakagawa T, Kang DH, Feig DI, Herrera-Acosta J. Subtle renal injury is likely a common mechanism for salt-sensitive essential hypertension. J. Hypertension 2005; 45: 326-330.

7 Wang TJ, Evans JC, Meigs JB, Rifai N, Fox CS, D'Agostino RB, Levy D, Vasan RS. Lowgrade albuminuria and the risks of hypertension and blood pressure progression. Circulation 2005; 111: 1370-1376.

8 Forman JP, Fisher ND, Schopick EL, Curhan GC. Higher levels of albuminuria within the normal range predict incident hypertension. J Am Soc Nephrol 2003; 19: 1983-1988.

9 Konta T, Hao Z, Abiko H, Ishikawa M, Takahashi T, Ikeda A, Ichikawa K, Takasaki S, Kubota I. Prevalence and risk factor analysis of microalbuminuria in Japanese general population: the Takahata study. Kidney Int 2006; 70: 751-756.

10 Levey AS, Eckardt KU, Tsukamoto Y, Levin A, Coresh J, Rossert J, De Zeeuw D, Hostetter TH, Lameire N, Eknoyan G. Definition and classification of chronic kidney disease: a position statement from kidney disease: Improving Global Outcomes (KDIGO). Kidney Int 2005; 67: 2089-2100.

11 Matsuo S, Imai E, Horio M, Yasuda Y, Tomita K, Nitta K, Yamagata K, Tomino Y, Yokoyama $\mathrm{H}$, Hishida A, Collaborators developing the Japanese equation for estimated GFR. Revised equations for estimated GFR from serum creatinine in Japan. Am J Kidney Dis 2009; 53: 982-992.

12 Kawasaki T, Itoh K, Uezono K, Sasaki H. A simple method for estimating 24-h urinary sodium and potassium excretion from second morning voiding urine specimen in adults. Clin Exp Pharmacol Physiol 1993; 20: 7-14.

13 Iwata K, Saito H, Moriyama M, Nakano A. Follow up study of renal tubular dysfunction and mortality in residents of an area polluted with cadmium. Br J Ind Med 1992; 49: 736-737.

14 Buckalew Jr VM, Berg RL, Wang SR, Porush JG, Rauch S, Schulman G. Prevalence of hypertension in 1,795 subjects with chronic renal disease: the modification of diet in renal disease study baseline cohort. Modification of Diet in Renal Disease Study Group. Am J Kidney Dis 1996; 28: 811-821.

15 Yuyun MF, Adler Al, Wareham NJ. What is the evidence that microalbuminuria is a predictor of cardiovascular disease events? Curr Opin Nephrol Hypertens 2005; 14: 271-276.

16 Cirillo M, Senigalliesi L, Laurenzi M, Alfieri R, Stamler J, Stamler R, Panarelli W, De Santo NG. Microalbuminuria in nondiabetic adults: relation of blood pressure, body mass index, plasma cholesterol levels, and smoking: the Gubbio Population Study. Arch Intern Med 1998; 158: 1933-1939.

17 Stehouwer CD, Fischer HR, Hackeng WH, den Ottolander GJ, Donker AJ. Diurnal variation in urinary protein excretion in diabetic nephropathy. Nephrol Dial Transplant 1991; 6: 238-243. 